\title{
Clinical implications of germline mutations in breast cancer: TP53
}

\author{
Katherine Schon $^{1} \cdot$ Marc Tischkowitz $^{1,2,3}$
}

Received: 23 December 2016 / Accepted: 3 October 2017 / Published online: 16 October 2017

(c) The Author(s) 2017. This article is an open access publication

\begin{abstract}
Purpose This review describes the prevalence of germline TP53 mutations, the risk of breast cancer and other cancers in mutation carriers and management implications for women with breast cancer and unaffected women.

Methods Literature review of English language papers available through PubMed.

Results Women who carry germline mutations in the TP53 gene have a very high risk of breast cancer of up to $85 \%$ by age 60 years. Most of these breast cancers are early onset with a median age at diagnosis of 34 years. Approximately $5-8 \%$ of women presenting with breast cancer under 30 years old have a germline TP53 gene mutation. Breast cancers in women with TP53 mutations are more likely to be hormone receptor positive and/or Her2 positive. Mastectomy is recommended over lumpectomy in TP53 mutation carriers who have breast cancer so that adjuvant breast radiotherapy can be avoided. Risk-reducing surgery should be considered due to the high contralateral breast cancer risk. Mutation carriers are at high risk of various childhood and adult-onset cancers with a very lifetime risk of malignancy, the commonest malignancies being breast cancer and soft tissue sarcoma. In unaffected female mutation carriers, MRI breast screening or risk-reducing surgery is recommended. The
\end{abstract}

Marc Tischkowitz

mdt33@cam.ac.uk

1 East Anglian Medical Genetics Service, Cambridge University Hospitals NHS Trust, Cambridge, UK

2 Department of Medical Genetics and National Institute for Health Research Cambridge Biomedical Research Centre, University of Cambridge, Cambridge, UK

3 Department of Medical Genetics, Addenbrooke's Treatment Centre, Cambridge Biomedical Campus, Box 238, Level 6, Cambridge CB2 0QQ, UK optimal surveillance for other cancers is currently unclear and should ideally be performed as part of a clinical trial. Conclusions Identifying a TP53 mutation in a gene panel test is a challenging result for the patient and clinician due to the high risk of second primaries and the lack of consensus aboutsurveillance.

Keywords TP53 $\cdot$ Gene panel testing $\cdot \mathrm{Li}-$ Fraumeni syndrome

\section{Introduction}

Breast cancer gene panel testing has become widely available for women who have been diagnosed with breast cancer and for unaffected women who are concerned about a strong family history of breast cancer. Mutations in the BRCAI and $B R C A 2$ genes remain by far the most common genetic explanation for a strong family history of breast cancer. Germline mutations in TP53 may cause an even higher risk of breast cancer, but these are much rarer than BRCA1/BRCA2 mutations.

The TP53 gene is a crucial tumour suppressor gene which has been called 'the guardian of the genome'. The cellular tumour antigen p53 protein acts as a checkpoint control following DNA damage. It either activates downstream genes to repair the damage or initiates apoptosis. Somatic mutations in TP53 occur very commonly in the formation of many cancer types and were found in $42 \%$ of samples from 12 different cancer types in the Pan-Cancer cohort [1], making it the most frequently mutated gene.

Germline mutations in the TP53 gene cause a familial cancer predisposition. The syndrome was first observed in 1969 by Li and Fraumeni who described four families of children with soft tissue sarcomas [2]. Mutation carriers 
have a very high lifetime risk of malignancy and the commonest cancers are soft tissue sarcomas and breast cancer in women. Depending on the pattern of cancers in a family, it may be described as having Li-Fraumeni Syndrome (LFS) (OMIM 151623), Li-Fraumeni-like syndrome (LFL) or it may not meet the diagnostic criteria for these. Various sets of diagnostic criteria and testing criteria have been developed (see table).

\begin{tabular}{cc}
\hline Classic Li-Fraumeni syndrome & All of the following \\
[3] & Proband with sarcoma diagnosed \\
before age 45 years \\
A first-degree relative with any \\
cancer before 45 years \\
A first or second-degree relative \\
with any cancer before 45 years \\
or a sarcoma at any age \\
All of the following \\
Proband with any childhood can- \\
cer, or a sarcoma, brain tumour \\
or ACC with onset < 45 years \\
A first or second-degree relative \\
with a core LFS cancer (sar- \\
coma, breast cancer, brain \\
tumour, ACC or leukaemia) with \\
onset at any age \\
A first or second-degree relative \\
with any cancer before age \\
60 years \\
Two first-degree or second-degree \\
relatives with core LFS malig- \\
nancies (sarcoma, premenopau- \\
sal breast cancer, brain tumour \\
ACC, leukaemia, lung [bron- \\
choalveolar] cancer) at any age \\
Proband with a cancer belonging \\
to the Li-Fraumeni spectrum \\
before age 46 AND at least one \\
first- or second-degree rela- \\
tive with a LFS tumour (except \\
breast cancer if proband has \\
breast cancer) before age 56 or \\
with multiple tumours \\
OR \\
Proband with multiple tumours, \\
two of which belong to LFS \\
spectrum and first before age \\
46 years \\
OR \\
Proband with adrenocortical carci- \\
noma or choroid plexus tumour, \\
regardless of family history \\
\hline Eeles [5] \\
\end{tabular}

\section{Prevalence of mutations}

\section{General population}

The frequency of germline pathogenic variants in TP53 in the general population is unknown and has been estimated using penetrance figures. The estimates vary between 1 in 5000 [7] and 1 in 20,000 [8]. Gonzalez and colleagues estimated the frequency of TP53 germline mutations in the general population using the frequency of specific cancers (breast cancer age $\leq 30$ years and adrenocortical carcinoma) in the general population and the frequency of that cancer being due to TP53 germline mutations. This gave an estimated frequency of 1 in 17,000 to 1 in 23,000 people. A recent study of germline variation in cancer-susceptibility genes in a healthy cohort found 15 TP53 missense variants and did not find any nonsense or frameshift variant in 681 individuals [9]; one missense variant was likely pathogenic and the others were variants of unknown clinical significance.

\section{Prevalence of mutations in women with early onset breast cancer}

The prevalence of TP53 mutations among women with early onset breast cancer has been studied in various populations $[10,11]$. McCuaig et al. estimated that $5-8 \%$ of women with breast cancer diagnosed under age 30 years and no pathogenic variant in $B R C A 1$ or $B R C A 2$ will have a pathogenic variant in TP53, and a smaller proportion of women with breast cancer diagnosed aged 30-39 years [12]. The likelihood of having a TP53 mutation is increased if there is a family history of LFS-related cancers, or a personal history of an additional LFS-related cancer.

In a series of patients who had a germline TP53 mutation ascertained due to having a young onset cancer, it was estimated that $7-20 \%$ of the mutations were de novo [13]. This is in contrast to hereditary breast and ovarian cancer syndrome due to $B R C A 1$ or $B R C A 2$ gene mutations, where de novo mutations are exceedingly rare. This observation supports testing very young onset breast cancer patients for $T P 53$, even in the absence of family history.

\section{Prevalence of TP53 mutations in women who have breast cancer gene panel testing}

The prevalence of TP53 mutations among women who have had panel testing is low at under $1 \%$ in four recent studies. Buys et al. found 61 women with TP53 mutations among 35,409 women with breast cancer who had testing using a panel of 25 cancer genes $(0.17 \%)$ [14]. Moran et al. detected one TP53 mutation among 190 breast cancer patients with a strong family history and previous negative $B R C A 1 / B R C A 2$ 
testing using a protein truncation test $(0.53 \%)$ [15]. Kapoor et al. found one TP53 mutation among 377 women who were offered gene testing by breast surgeons using multigene panels (5-43 genes, average 14.7) (0.27\%) [16]. Susswein et al. reported results for over 10,000 consecutive cases referred for evaluation of germline cancer genes. They reported nine pathogenic and one likely pathogenic TP53 mutation among 3315 women with breast cancer who had not had previous $B R C A 1 / B R C A 2$ testing $(0.30 \%)$, and three pathogenic and one likely pathogenic TP53 mutation among 1894 women with breast cancer who had previous BRCA1/BRCA2 testing $(0.21 \%)[17]$.

\section{Founder mutations}

There is a high prevalence of the c.1010G > A, p.(Arg337His) mutation in exon 10 (often referred to as R337H) in Southeast and Southern Brazil [18]. Individuals with this mutation have a similar lifetime cancer prevalence to other LFS carriers (about 90\%) but a lower penetrance at young ages $(<20 \%$ at age 30 years, compared to $50 \%$ in other LFS carriers) [19]. It has been suggested that women affected with breast cancer under the age of 45 years in Southeast and Southern Brazil should be offered testing for this mutation, irrespective of family history [20]. The $\mathrm{R} 337 \mathrm{H}$ mutation is not common among women diagnosed with breast cancer in Portugal [21]. We are not aware of any other founder mutations in the TP53 gene.

\section{Penetrance of breast cancer}

The penetrance of breast cancer in women with TP53 mutations is very high with a cumulative incidence of $85 \%$ by age 60 years in the National Cancer Institute Li-Fraumeni Syndrome cohort [22]. The annual hazard for female breast cancer started to increase in the late teens and peaked at approximately 40 years. This was a highly selected cohort of 286 individuals from 107 families. Most met the criteria for LFS (43\%) or Li-Fraumeni-like syndrome (38\%). $8 \%$ had $\geq 3$ primaries and $10 \%$ had tested positive for a TP53 mutation without meeting any of the current diagnostic criteria or testing guidelines. Another study found that the median age of onset of breast cancer was 34 years [23]. The lifetime risks in individuals without a strong family history might be lower.

\section{Range of cancer sites implicated}

The overall lifetime risk of cancer in individuals with TP53 mutations is very high. Wu et al. estimated that the cancer-free survival probabilities for female TP53 mutation carriers were $65.2,33.0$ and $2.9 \%$ at ages 30,45 and 60 years, respectively. The corresponding cancer-free survival rates for male carriers were $83.4,62.5$ and $22.2 \%$ [24]. This gender difference is primarily the result of the high incidence of breast cancer among women with LFS.

There are a wide variety of cancer sites implicated in LFS. LFS was originally described in the families of children with soft tissue sarcomas [2], the second commonest cancer diagnosis (after breast cancer) in the National Cancer Institute Li-Fraumeni Syndrome cohort [22]. The 'core' cancers described were sarcomas, breast cancer, adrenocortical cancers and brain tumours. In a cohort of 525 patients tested, 9/9 patients with choroid plexus tumours had an identifiable TP53 mutation [8]. Other cancers become commoner in older TP53 mutation carriers, such as lung, colorectal and prostate cancer. Leukaemias and lymphomas can also occur but are not cardinal features.

There is a high risk for multiple primary cancers and a study of 200 individuals from 24 LFS families found that $15 \%$ of individuals developed a second cancer, $4 \%$ had a third cancer and $2 \%$ had a fourth. The cumulative probability of a second cancer occurrence at 30 years after the first cancer was estimated as $75 \%( \pm 10 \%)$ [25].

Ruijs and colleagues provide estimates for the relative risk of different cancer types in LFS [26]. They found that the highest relative risks were for bone cancers (RR 107, 95\% confidence intervals 49-203), connective tissue cancers (RR 61, CI 33-102) and brain tumours (RR 35, CI 19-60). The relative risk for breast cancer was 6.4 (95\% confidence intervals 4.3-9.3).

\section{Determining the pathogenicity of variants}

The TP53 gene is located on chromosome 17p13.1 and encodes the cellular tumour antigen $\mathrm{p} 53$. The gene has 11 exons and is $20 \mathrm{~kb}$ in genomic length. The coding region encompasses exons $2-11$ while exon 1 is non-coding and contains two transcriptional start sites. The majority of pathogenic variants are missense variants $73 \%$ in the study by Olivier and colleagues [23]) or small 1-4 bp deletions [23]. There are mutational hot spots at codons 133, 175, 213, 220, 245, 248, 273, 282 and 337 [27]. These codons are also mutational hotspots in sporadic tumours. Many of these are within exons 5-8 which encode the core DNA-binding region of the gene.

$95 \%$ of mutations can be detected by sequence analysis of all exons [28]. Only approximately $1 \%$ of mutations are deletions or duplications involving the coding region, exon 1 or the promoter [28]. A functional assay may be useful in determining the clinical significance of novel pathogenic 
missense variants but this is only performed in certain research laboratories.

There are several databases with curated information on TP53 variants, including the p53 Mutation in Human Cancer (http://p53.free.fr/) and the IARC TP53 Database (http:// p53.iarc.fr). A recent paper by Bouaoun [27] used the IARC database to provide an update on TP53-inherited variants, including those that should be considered as neutral frequent variants.

\section{Genotype-phenotype correlations}

There have been several studies looking at genotype-phenotype correlations in families with LFS and LFS-like syndrome. Birch and colleagues' study of 34 families (20 LFS and 14 LFL) showed that individuals with missense mutations in the DNA-binding region had higher overall rates of cancer with significantly higher rates of breast cancer and central nervous system tumours compared to individuals with missense mutations in other parts of the gene or protein-truncating mutations [29]. A study by Olivier et al. analysed the IARC database (including 1068 individuals from 265 families) and found that missense mutations outside the DNA-binding region are more commonly associated with adrenocortical carcinoma compared to missense mutations in the DNA-binding domain. They also noted that individuals with missense mutations in the DNA-binding domain were more likely to have early onset breast cancer compared to those with missense mutations outside the DNA-binding domain (32 years vs. 42 years) and that mutations leading to a TP53 null phenotype are associated with earlier onset brain tumours [23].

\section{Management implications}

\section{Management of risk of breast cancer}

The option for risk-reducing bilateral mastectomy or breast screening should be considered in women without cancer with a mutation in the TP53 gene. In the UK, annual MRI breast screening is recommended from age 20 to 49 years and should be considered between 50 and 69 years. Mammography is not recommended [30]. In the USA, National Comprehensive Cancer Network guidelines recommend annual breast MRI 20-29 years and annual MRI and mammography from 30 to 75 years [31]. In Australia, national guidelines recommend that bilateral mastectomy should be offered, otherwise annual breast MRI is recommended from 20 to 50 years [32]. Based on the finding that breast cancer risk increases significantly after the second decade [22], bilateral mastectomy should be considered from age 20 (in line with NCCN guidelines [31]). The annual breast cancer risk peaks at around age 40-45 and then decreases [22]. Bilateral mastectomy is less likely to benefit women over 60 years.

\section{Management of breast cancer}

Mastectomy rather than lumpectomy is recommended to reduce the risks of a second primary breast cancer and to avoid radiotherapy where possible. Bilateral mastectomy should also be considered due to the risk of a contralateral breast cancer. Contralateral breast cancer risk will depend on the patient's age, but there are no clear figures from the literature.

There are concerns about increased risk of radiationinduced second primary tumours. Heymann et al. [33] describe a series of 8 patients with germline TP53 mutations who were treated for breast cancer between 1997 and 2007 from 47 documented Li-Fraumeni families; three underwent conservative breast surgery with post-operative radiotherapy, three had mastectomy and radiotherapy and two had mastectomy with no radiotherapy. Among the six who received radiotherapy, there were three ipsilateral breast recurrences, three contralateral breast cancers, two radio-induced cancers (one breast histiocytoma fibrosarcoma and one chest wall angiosarcoma) and three new primaries (including one papillary thyroid carcinoma which developed inside the radiation field after 2 years). One contralateral breast cancer occurred in the two patients who did not have radiotherapy, with a median follow-up of 6 years. Despite the small sample size, this study does suggest that radiotherapy should be avoided or used with extreme caution in TP53 mutation carriers after very careful consideration of the risks and benefits.

A study of the breast cancer histopathology in TP53 carriers showed that most invasive ductal carcinomas and ductal carcinomas in situ (DCIS) in LFS are hormone receptor positive and/or HER-2 positive [34]. In this study, there were 32 invasive ductal carcinomas in 30 women with confirmed germline TP53 mutations. $84 \%$ of the tumours stained positive for oestrogen receptors, $72 \%$ for progesterone receptors and 63\% showed HER-2 amplification and/or overexpression. Melhem-Bertrandt et al. [35] have also reported that women who are TP53 mutation carriers are more likely to have HER2 amplification and/ or overexpression (present in $67 \%$ of cases and $25 \%$ of controls in this study).

These histopathological findings have management implications as treatment is more likely to include Trastuzumab (Herceptin) and hormone therapy. There are no published data on treatment response to chemotherapeutic agents so standard chemotherapy regiments should be used. 


\section{Management of risks of other cancers}

There is no international consensus about the best surveillance for TP53 mutation carriers. In the UK, breast MRI screening is the only recommendation. In the USA, annual complete physical examination including neurologic and skin examination is recommended, and 2-5 yearly colonoscopy from age 25 [31]. In the Netherlands, annual breast surveillance from age 20 to 25 and an optional annual physical examination is recommended [36]. The Australian guidelines recommend annual clinical review, and to consider 2-5 yearly colonoscopy from 25 years if there is a family history of colorectal cancer, or 2-5 yearly endoscopy if there is a family history of gastric cancer [32].

An intensive and comprehensive surveillance programme has been proposed by Villani et al. for children and adults who carry a TP53 germline mutation [37]. This uses multiple modalities (physical examination, blood tests, ultrasound, mammography, MRI and colonoscopy). The results of a non-randomised trial showed good long-term compliance with the protocol. It reported a significantly improved five-year overall survival in the surveillance group. However, there was a very high rate of symptomatic tumours in the group who initially declined surveillance. A larger-scale randomised controlled trial is required to evaluate the protocol further.

Neonatal testing for the c.1010G > A, p.(Arg337His) mutation and subsequent surveillance for adrenocortical tumours in mutation carriers has been evaluated in a large, non-randomised clinical trial in Southern Brazil [38]. Adrenocortical tumours diagnosed in the surveillance group were smaller and had an improved clinical outcome. Although the results are not directly transferrable to other populations of TP53 carriers with different mutations, it does suggest that surveillance for adrenocortical tumours in children should be considered.

The UK SIGNIFY study [39] investigated the role of oneoff whole body non-contrast MRI screening in asymptomatic TP53 mutation carriers. Four malignancies were diagnosed among 44 TP53 mutation carriers and none in matched controls. Two malignancies were not picked up on scan-one patient developed leukaemia and one became symptomatic from a mediastinal liposarcoma which was thought to be a pericardial cyst on scan. The difference between cancer detection in carriers and controls was not statistically significant, but the trend of the results suggests that whole body MRI is a useful investigation.

\section{Ongoing clinical trials}

There are several ongoing trials using whole body MRI screening (reviewed in McBride 2014 [40] and Ballinger 2015 [41]). The LIFSCREEN trial [42] at the Institut
Gustave Roussy (Villejuif, France) is a randomised trial with annual whole body MRI screening for three years in the intervention arm and standard care in the control arm. It is recruiting individuals with TP53 mutations (age 5-71 years). The Surveillance of Multi-Organ Cancer Prone Syndrome (SMOC) trial [43] in Australia is enrolling individuals with a germline mutation in a cancer risk gene (including TP53) and those at $50 \%$ risk aged $18-70$ years for a period of 3 years. It is a non-randomised trial using annual physical examination, full blood count, whole body MRI, breast MRI, ultrasound and mammography (in females) with additional procedures of gastroscopy and colonoscopy based on family history. The Dana Faber Cancer Institute whole body MRI study involves annual physical examination and whole body MRI and is currently recruiting children only.

Based on the observation that metformin has been associated with reduced cancer risk in several epidemiological studies [44], a pilot study for chemoprevention using metformin has been commenced which assesses the safety and tolerability of the drug over 14 weeks, measuring IGF-1, insulin and IGFBP3 levels in blood at baseline, and weeks 0 and 8 [45].

As the optimal screening for other cancers in TP53 mutation carriers remains unclear, screening should ideally be undertaken as part of clinical trial.

\section{Example}

A fifty-year old unaffected woman with no family history has a TP53 pathogenic variant detected on panel testing. The evidence is that this scenario will be very unusual, so we would advise reviewing the literature about the pathogenicity of the specific variant, and we would consider repeating the test (in case of a sample error). We would advise that she is at high risk of developing breast cancer, although she has 'lived through' a substantial part of that risk. We would advise annual MRI breast screening or bilateral mastectomy. We would also consider a baseline whole body MRI, based on the UK SIGNIFY study results.

\section{Support groups}

Some women may require additional psychological support. There are several patient support groups including the Li-Fraumeni Syndrome Association <http://www.lfsassociation.org $>$ based in the USA, and the George Pantziarka TP53 Trust <http://www.tp53.co.uk> based in the UK.

\section{Conclusions}

Finding a germline TP53 mutation in a woman with breast cancer has significant clinical implications for the patient 
and her family. Mastectomy is preferred in the treatment of breast cancer so that radiotherapy can be avoided or minimised. Risk-reducing contralateral mastectomy should also be considered, otherwise MRI breast screening should be initiated. The risk of developing second or third primary malignancies is high, and optimal surveillance is currently unclear. Where possible, screening should be undertaken as part of a clinical trial.

Acknowledgements KS holds an Academic Clinical Fellowship funded by the National Institute for Health Research (3090). MT is funded by the European Union Seventh Framework Program (20072013)/European Research Council (310018).

Open Access This article is distributed under the terms of the Creative Commons Attribution 4.0 International License (http://creativecommons.org/licenses/by/4.0/), which permits unrestricted use, distribution, and reproduction in any medium, provided you give appropriate credit to the original author(s) and the source, provide a link to the Creative Commons license, and indicate if changes were made.

\section{References}

1. Kandoth C, McLellan MD, Vandin F, Ye K, Niu B, Lu C et al (2013) Mutational landscape and significance across 12 major cancer types. Nature 502(7471):333-339

2. Li FP, Fraumeni JF (1969) Soft-tissue sarcomas, breast cancer, and other neoplasms. A familial syndrome? Ann Intern Med 71(4):747-752

3. Li FP, Fraumeni JF, Mulvihill JJ, Blattner WA, Dreyfus MG, Tucker MA et al (1988) A cancer family syndrome in twentyfour kindreds. Cancer Res 48(18):5358-5362

4. Birch JM, Hartley AL, Tricker KJ, Prosser J, Condie A, Kelsey AM et al (1994) Prevalence and diversity of constitutional mutations in the p53 gene among $21 \mathrm{Li}-$ Fraumeni families. Cancer Res 54(5):1298-1304

5. Eeles RA (1995) Germline mutations in the TP53 gene. Cancer Surv 25:101-124

6. Tinat J, Bougeard G, Baert-Desurmont S, Vasseur S, Martin C, Bouvignies E et al (2009) 2009 version of the Chompret criteria for Li Fraumeni syndrome. J Clin Oncol 27(26):e108-e109 author reply e110

7. Lalloo F, Varley J, Ellis D, Moran A, O'Dair L, Pharoah P et al (2003) Prediction of pathogenic mutations in patients with early-onset breast cancer by family history. Lancet Lond Engl 361(9363):1101-1102

8. Gonzalez KD, Noltner KA, Buzin CH, Gu D, Wen-Fong CY, Nguyen VQ et al (2009) Beyond Li Fraumeni syndrome: clinical characteristics of families with p53 germline mutations. J Clin Oncol 27(8):1250-1256

9. Bodian DL, McCutcheon JN, Kothiyal P, Huddleston KC, Iyer RK, Vockley JG et al (2014) Germline variation in cancer-susceptibility genes in a healthy, ancestrally diverse cohort: implications for individual genome sequencing. PLoS ONE 9(4):e94554

10. Lee DSC, Yoon S-Y, Looi LM, Kang P, Kang IN, Sivanandan $\mathrm{K}$ et al (2012) Comparable frequency of BRCA1, BRCA2 and TP53 germline mutations in a multi-ethnic Asian cohort suggests TP53 screening should be offered together with BRCA1/2 screening to early-onset breast cancer patients. Breast Cancer Res BCR 14(2):R66
11. Mouchawar J, Korch C, Byers T, Pitts TM, Li E, McCredie MRE et al (2010) Population-based estimate of the contribution of TP53 mutations to subgroups of early-onset breast cancer: Australian breast cancer family study. Cancer Res 70(12):4795-4800

12. McCuaig JM, Armel SR, Novokmet A, Ginsburg OM, Demsky R, Narod SA et al (2012) Routine TP53 testing for breast cancer under age 30: ready for prime time? Fam Cancer 11(4):607-613

13. Gonzalez KD, Buzin CH, Noltner KA, Gu D, Li W, Malkin D et al (2009) High frequency of de novo mutations in Li-Fraumeni syndrome. J Med Genet 46(10):689-693

14. Buys SS, Sandbach JF, Gammon A, Patel G, Kidd J, Brown KL et al (2017) A study of over 35,000 women with breast cancer tested with a 25 -gene panel of hereditary cancer genes. Cancer 123(10):1721-1730

15. Moran O, Nikitina D, Royer R, Poll A, Metcalfe K, Narod SA et al (2016) Revisiting breast cancer patients who previously tested negative for BRCA mutations using a 12-gene panel. Breast Cancer Res Treat. doi:10.1007/s10549-016-4038-y

16. Kapoor NS, Curcio LD, Blakemore CA, Bremner AK, McFarland RE, West JG et al (2015) Multigene panel testing detects equal rates of pathogenic BRCA1/2 Mutations and has a higher diagnostic yield compared to limited BRCA1/2 analysis alone in patients at risk for hereditary breast cancer. Ann Surg Oncol 22(10):3282-3288

17. Susswein LR, Marshall ML, Nusbaum R, Vogel Postula KJ, Weissman SM, Yackowski L et al (2016) Pathogenic and likely pathogenic variant prevalence among the first 10,000 patients referred for next-generation cancer panel testing. Genet Med 18(8):823-832

18. Achatz MI, Zambetti GP (2016) The inherited p53 mutation in the Brazilian population. Cold Spring Harb Perspect Med. doi:10.1101/cshperspect.a026195

19. Malkin D (2011) Li-Fraumeni syndrome. Genes Cancer 2(4):475-484

20. Andrade KC, Santiago KM, Fortes FP, Mambelli LI, Nóbrega AF, Achatz MI (2016) Early-onset breast cancer patients in the South and Southeast of Brazil should be tested for the TP53 p. R337H mutation. Genet Mol Biol 39(2):199-202

21. Giacomazzi J, Correia RL, Palmero EI, Gaspar JF, Almeida M, Portela $\mathrm{C}$ et al (2014) The Brazilian founder mutation TP53 p. $\mathrm{R} 337 \mathrm{H}$ is uncommon in Portuguese women diagnosed with breast cancer. Breast J 20(5):534-536

22. Mai PL, Best AF, Peters JA, DeCastro RM, Khincha PP, Loud JT et al (2016) Risks of first and subsequent cancers among TP53 mutation carriers in the National Cancer Institute Li-Fraumeni syndrome cohort. Cancer 122:3673-3681

23. Olivier M, Goldgar DE, Sodha N, Ohgaki H, Kleihues P, Hainaut $\mathrm{P}$ et al (2003) Li-Fraumeni and related syndromes: correlation between tumor type, family structure, and TP53 genotype. Cancer Res 63(20):6643-6650

24. Wu C-C, Krahe R, Lozano G, Zhang B, Wilson CD, Jo E-J et al (2011) Joint effects of germ-line TP53 mutation, MDM2 SNP309, and gender on cancer risk in family studies of Li-Fraumeni syndrome. Hum Genet 129(6):663-673

25. Hisada M, Garber JE, Fung CY, Fraumeni JF, Li FP (1998) Multiple primary cancers in families with Li-Fraumeni syndrome. $\mathrm{J}$ Natl Cancer Inst 90(8):606-611

26. Ruijs MWG, Verhoef S, Rookus MA, Pruntel R, van der Hout AH, Hogervorst FBL et al (2010) TP53 germline mutation testing in 180 families suspected of Li-Fraumeni syndrome: mutation detection rate and relative frequency of cancers in different familial phenotypes. J Med Genet 47(6):421-428

27. Bouaoun L, Sonkin D, Ardin M, Hollstein M, Byrnes G, Zavadil $\mathrm{J}$ et al (2016) TP53 variations in human cancers: new lessons from the IARC TP53 database and genomics data. Hum Mutat 37(9):865-876 
28. Schneider K, Zelley K, Nichols KE, Garber J. Li-Fraumeni Syndrome (1993). In: Pagon RA, Adam MP, Ardinger HH, Wallace SE, Amemiya A, Bean LJ, et al., editors. GeneReviews $\left({ }^{\circledR}\right)$. Seattle (WA): University of Washington, Seattle. http://www.ncbi.nlm. nih.gov/books/NBK1311/. Accessed 5 Dec 2016

29. Birch JM, Blair V, Kelsey AM, Evans DG, Harris M, Tricker KJ et al (1998) Cancer phenotype correlates with constitutional TP53 genotype in families with the Li-Fraumeni syndrome. Oncogene 17(9):1061-1068

30. Familial breast cancer: classification, care and managing breast cancer and related risks in people with a family history of breast cancer I 1-Recommendations I Guidance and guidelines I NICE. https://www.nice.org.uk/guidance/cg164/chapter/1-recommendations. Accessed 1 Dec 2016

31. National Comprehensive Cancer Network (2014) Genetic/familial high risk assessment: breast and ovarian. Li Fraumeni syndrome management. NCCN Clinical Practice Guidelines in Oncology; Version 2. 2014

32. eviQ Cancer Treatments Online (2016) Risk management for LiFraumeni syndrome. http://www.eviq.org.au. Accessed 12 Dec 2016

33. Heymann S, Delaloge S, Rahal A, Caron O, Frebourg T, Barreau L et al (2010) Radio-induced malignancies after breast cancer postoperative radiotherapy in patients with $\mathrm{Li}-$ Fraumeni syndrome. Radiat Oncol Lond Engl 5:104

34. Masciari S, Dillon DA, Rath M, Robson M, Weitzel JN, Balmana J et al (2012) Breast cancer phenotype in women with TP53 germline mutations: a Li Fraumeni syndrome consortium effort. Breast Cancer Res Treat 133(3):1125-1130

35. Melhem-Bertrandt A, Bojadzieva J, Ready KJ, Obeid E, Liu DD, Gutierrez-Barrera AM et al (2012) Early onset HER2-positive breast cancer is associated with germline TP53 mutations. Cancer 118(4):908-913

36. Lammens CRM, Bleiker EMA, Aaronson NK, Wagner A, Sijmons RH, Ausems MGEM et al (2010) Regular surveillance for
Li-Fraumeni syndrome: advice, adherence and perceived benefits. Fam Cancer 9(4):647-654

37. Villani A, Shore A, Wasserman JD, Stephens D, Kim RH, Druker $\mathrm{H}$ et al (2016) Biochemical and imaging surveillance in germline TP53 mutation carriers with Li-Fraumeni syndrome: 11 year follow-up of a prospective observational study. Lancet Oncol 17(9):1295-1305

38. Custódio G, Parise GA, Kiesel Filho N, Komechen H, Sabbaga CC, Rosati R et al (2013) Impact of neonatal screening and surveillance for the TP53 R337H mutation on early detection of childhood adrenocortical tumors. J Clin Oncol 31(20):2619-2626

39. Saya S, Killick E, Thomas S, Taylor N, Bancroft EK, Rothwell J et al (2017) Baseline results from the UK SIGNIFY study: a whole-body MRI screening study in TP53 mutation carriers and matched controls. Fam Cancer 16:433

40. McBride KA, Ballinger ML, Killick E, Kirk J, Tattersall MHN, Eeles RA et al (2014) Li-Fraumeni syndrome: cancer risk assessment and clinical management. Nat Rev Clin Oncol 11(5):260-271

41. Ballinger ML, Mitchell G, Thomas DM (2015) Surveillance recommendations for patients with germline TP53 mutations. Curr Opin Oncol 27(4):332-337

42. US National Libary of Medicine. ClinicalTrials.gov (2014). http:// clinicaltrials.gov/show/NCT01464086. Accessed 13 Dec 2016

43. ANZCTR: Australia New Zealand Clinical Trials Registry. Trial from ANZCTR (2013). http://www.ANZCTR.org.au/ ACTRN12613000987763.aspx. Accessed 13 Dec 2016

44. Gandini S, Puntoni M, Heckman-Stoddard BM, Dunn BK, Ford L, DeCensi A et al (2014) Metformin and cancer risk and mortality: a systematic review and meta-analysis taking into account biases and confounders. Cancer Prev Res 7(9):867-885

45. US National Libary of Medicine. ClinicalTrials.gov (2013). http:// clinicaltrials.gov/show/NCT01981525. Accessed 13 Dec 2016 